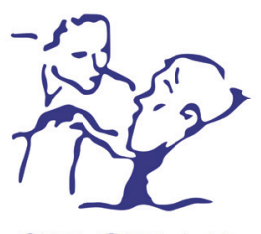

Medicina Paliativa

www.medicinapaliativa.es

ORIGINAL BREVE

\title{
¿Cómo es la atención médica urgente a pacientes en seguimiento por un equipo de soporte domiciliario?
}

\author{
Ana Espejo González*1, María Elena Baeza Monedero², Jana Albéniz López³, \\ Antonio Sacristán Rodea ${ }^{4}$, Gloria Galdón Planas ${ }^{4}$, Sergio Collazo Carrera ${ }^{4}$ y \\ Manuel González Torrejón ${ }^{4}$
}

${ }^{1}$ Centro de Salud Jazmín, Madrid, España. ${ }^{2}$ Servicio de Geriatría, Hospital Universitario Rey Juan Carlos, Móstoles, Madrid, España. ${ }^{3}$ Servicio de Geriatría, Hospital Universitario Ramón y Cajal, Madrid, España. ${ }^{4}$ Equipo de Soporte Domiciliario del Área Este del SERMAS, Madrid, España

Recibido el 12 de febrero de 2018

Aceptado el 7 de mayo de 2018

\begin{abstract}
PALABRAS CLAVE
Medicina paliativa, cuidados

domiciliarios, cuidados urgentes, síntomas.
\end{abstract}

\begin{abstract}
Resumen
Objetivos: Conocer los motivos de consulta urgente de los pacientes en seguimiento por un equipo de soporte de atención domiciliaria (ESAD), así como describir el perfil de los mismos, el horario de las consultas urgentes realizadas durante el periodo de seguimiento, la intervención llevada a cabo, la resolución de la misma y el lugar de fallecimiento.

Material y métodos: Estudio descriptivo, observacional, prospectivo y unicéntrico. De los pacientes derivados al ESAD del Área Este del SERMAS (Servicio Madrileño de Salud) en el periodo de estudio (de febrero a mayo de 2017), se incluyeron aquellos que habían realizado al menos una consulta urgente, bien acudiendo al Servicio de Urgencias del Hospital Ramón y Cajal (HRyC) o consultando telefónicamente al PAL-24 (servicio de atención telefónica 24 horas). Se realizó un seguimiento de 3 meses a partir del momento de la inclusión.

Resultados: De los 279 pacientes derivados al ESAD, 93 fueron incluidos en nuestro estudio, registrándose un total de 151 consultas. De ellos, 74 contactaron telefónicamente con el PAL-24 y 77 visitaron el Servicio de Urgencias del HRyC, en ambos casos por mal control de la disnea como síntoma principal (29,7 \% en PAL-24 y 30,3\% en Urgencias), seguido por dolor (12,2 \% en PAL-24 y $18,4 \%$ en Urgencias). La edad media fue de 78,8 años y la enfermedad de base más frecuente fue el carcinoma de pulmón $(21,5 \%)$, seguido del cáncer colorrectal. La atención urgente se realizó fuera del horario del ESAD en el 91,85\% de las llamadas al PAL-24 y en el $73,7 \%$ de las visitas a Urgencias. El PAL-24 resolvió telefónicamente el 48,6\% de las llamadas
\end{abstract}

\footnotetext{
"Autor para correspondencia:

Ana Espejo González

Centro de Salud Jazmín, c/ Jazmín 33, 28033 Madrid

Correo electrónico: aespegon@gmail.com
} 
y empleó opioides en el $27,5 \%$ de los casos, ansiolíticos en el 13,5\% y antiinflamatorios no esteroideos (AINE) en el 9,5\%. Durante el seguimiento, de los 76 pacientes que fallecieron $(81,7 \%)$, el $32,3 \%$ lo hizo en el domicilio, el $21,5 \%$ en una Unidad de Cuidados Paliativos, el 20,4 \% hospitalizados y el 7,5\% en Urgencias.

Conclusiones: El motivo más frecuente de consulta urgente es la disnea, seguida del mal control del dolor. En su mayoría son pacientes oncológicos, atendidos fuera del horario del ESAD. El perfil del paciente que representa nuestra muestra es el de un paciente de unos 78 años, con una enfermedad oncológica en fase terminal, en seguimiento por un equipo de ESAD. Un tercio del total de los pacientes termina falleciendo en el domicilio.

\section{KEYWORDS}

Palliative care, home

care, emergency

care, symptoms.

\section{Abstract}

Objectives: To assess the reasons for urgent consultations in patients followed by a home care support team (ESAD in Spanish), and to describe patient profile, emergency consultation timing, interventions performed and their outcome, and place of demise.

Methods: A descriptive, observational, prospective, single-center study. Of all patients in the ESAD program from February to May 2017, those with at least one emergency visit to the Emergency Room at Hospital Ramón y Cajal (HRyC) or an emergency telephone call to PAL-24 (24-hour telephone service) were included. A 3-month follow-up was performed.

Results: Of a total of 279 patients included in the ESAD program only 93 were enrolled in the study. A total of 151 urgent consultations were recorded, 74 calls to the PAL-24 service and 77 visits to the emergency room at HRyC, with dyspnea being the main symptom $(29.7 \%$ in PAL-24 calls and $30.3 \%$ in ER visits) followed by pain (12.2\% and $18.4 \%$, respectively). Mean age was 78.8 years and the most common diagnosis was lung cancer $(21.5 \%)$, followed by colorectal cancer. Urgent consultations were mainly needed outside ESAD working hours $(91.85 \%$ of calls to the PAL24 service and $73.7 \%$ of visits to the ER). The PAL-24 service telephonically solved $48.6 \%$ of calls by prescribing opioids in $27.5 \%$, anxiolytics in $13.5 \%$, and anti-inflammatory agents in $9.5 \%$ of cases. Following emergency care at the ER, $55.5 \%$ of patients were discharged back to home whereas $38.2 \%$ needed hospitalization and $6.6 \%$ died. During follow-up $76(81.7 \%)$ patients died $-32.3 \%$ at home, $21.5 \%$ at a palliative care unit, $20.4 \%$ at the hospital, and $7.5 \%$ in the emergency room.

Conclusions: The main reason for emergency care seeking was dyspnea followed by pain. Most patients had a cancer diagnosis and required emergency care outside the regular ESAD time schedule. The standard patient profile in our sample was 78 years of age, with end-stage cancer, followed by an ESAD team. One third of patients died at home.

Espejo González A, Baeza Monedero ME, Albéniz López J, Sacristán Rodea A, Galdón Planas G, Collazo Carrera S,González Torrejón M. ¿Cómo es la atención médica urgente a pacientes en seguimiento por un equipo de soporte domiciliario? Med Paliat. 2019;26(2):150-155.

\section{Introducción}

La atención domiciliaria proporciona cuidados a pacientes crónicos, terminales e inmovilizados, y su organización depende de las Gerencias de Atención Primaria.

En 1998, en la Comunidad de Madrid se creó un proyecto piloto denominado "Equipo de Soporte en Atención Domiciliaria" (ESAD), destinado a apoyar, formar y asesorar a los profesionales de Atención Primaria con el fin de dar una buena calidad en la atención domiciliaria'. En 1999 se creó el Programa de Atención Domiciliaria con Equipo de Soporte; posteriormente, en 2007 se puso en marcha el Plan Integral de Cuidados Paliativos de la Comunidad de Madrid 20052008, siendo en 2008 cuando se llevó a cabo la coordinación regional de los cuidados paliativos ${ }^{2}$. No es hasta 2010 cuando se implanta el Plan Estratégico de Cuidados Paliativos de la Comunidad de Madrid ${ }^{2}$. Dentro de las acciones concretas que recoge el Plan, se puso en marcha un servicio telefónico que atiende específicamente a pacientes de cuidados paliativos de forma permanente, centralizada para toda la región, 24 horas al día y 365 días al año. El servicio está integrado por personal médico y de enfermería, siendo accesible a través del 061, centro coordinador del SUMMA 112. Esta nueva plataforma se llamó PAL-24 y su objetivo es proporcionar asesoramiento, derivación a centro y, si es necesario, movilización de recursos sanitarios.

En la actualidad, en España existen un total de 417 equipos de cuidados paliativos, de los que 168 (40,2 \%) son ESAD. Su distribución geográfica abarca todas las comunidades autónomas ${ }^{2}$. Sin embargo, la transferencia de competen- 
cias en materia de sanidad a las comunidades autónomas ha contribuido a un desarrollo desigual en todo el país.

Existen diversos estudios, sobre todo europeos y americanos, que describen el manejo de las situaciones de urgencia en el ámbito de los cuidados paliativos y que reclaman la necesidad de realizar más investigaciones sobre este tema. A nivel nacional existen pocos estudios y presentan grandes limitaciones, fundamentalmente tamaños muestrales muy reducidos.

Algunos estudios recientes describen que el $75 \%$ de los pacientes mayores de 65 años visitó un Servicio de Urgencias Hospitalario en los últimos 6 meses de vida y el $51 \%$ de los pacientes en el último mes de vida ${ }^{3}$. También se han evaluado las visitas a Urgencias en los últimos 90 días previos al fallecimiento, obteniéndose que el $65,8 \%$ de los pacientes con tumores malignos visitó un Servicio de Urgencias Hospitalario en el último año de vida y un $47 \%$ de los pacientes en los 90 días previos a la muerte ${ }^{4}$.

Tras analizar el contexto sociosanitario descrito, nos planteamos como objetivo primario de nuestro estudio conocer los motivos de consulta urgente de los pacientes incluidos en el ESAD del Área Este del SERMAS y, como objetivos secundarios, describir el perfil de dichos pacientes, el horario de las consultas urgentes realizadas durante el periodo de seguimiento, la intervención llevada a cabo, la resolución de la misma y el lugar de fallecimiento.

\section{Métodos}

Se trata de un estudio descriptivo, observacional, prospectivo y unicéntrico.

Los criterios de inclusión fueron:

- Pacientes incluidos en el programa de cuidados paliativos domiciliarios (mayores de 18 años, en fase terminal [enfermedad en progresión sin posibilidad de tratamiento específico con mal pronóstico a corto plazo], con domicilio estable y presencia de cuidador), en seguimiento por el ESAD del sector Este del SERMAS durante los meses de febrero a mayo de 2017.

Registro de una consulta urgente al menos, entendiendo como consulta urgente aquella realizada a demanda del paciente o familia a recursos distintos del ESAD (en nuestro caso, acudiendo a Urgencias del HRyC o consultando telefónicamente al PAL-24).

Los criterios de exclusión fueron:

- Ausencia de consulta urgente durante los 3 meses de seguimiento.

Ausencia de registro electrónico o en papel de la consulta urgente.

Las variables analizadas fueron recogidas mediante tres formularios. El primero de ellos incluye los datos sociodemográficos (género, edad y cuidador principal), enfermedad de base o motivo principal por el que el paciente es derivado al ESAD y síntomas de la primera visita en domicilio (dolor, disnea, anorexia, etc.). En un segundo formulario se recoge la visita al HRyC (horario, motivo de consulta, última visita del ESAD, pruebas diagnósticas realizadas, tratamiento administrado y destino al alta). $Y$ en el tercero se registra la actuación del PAL-24 (horario, motivo de consulta, última visita del ESAD, fármacos utilizados, recursos movilizados y destino al alta).
Estos datos fueron extraídos durante la primera visita en el domicilio de los pacientes y mediante la consulta electrónica de programas informáticos como AP Madrid y Horus, sistemas informáticos de la red sanitaria de la Comunidad de Madrid.

La investigación fue aprobada por el Comité de Ética y el Comité de Ensayos Clínicos e Investigación del Área Este del SERMAS, obteniéndose la autorización de los individuos incluidos en el estudio.

El análisis estadístico se realizó mediante el programa estadístico SPSS versión 15.0, calculándose las frecuencias y porcentajes para las variables cualitativas, y las medias, desviaciones estándar o típicas, valores máximos y mínimos para las mediciones cuantitativas.

\section{Resultados}

Un total de 279 pacientes fue derivado al equipo de ESAD durante el periodo de febrero a mayo de 2017. De ellos, únicamente 93 cumplieron los criterios de inclusión, bien acudiendo a Urgencias directamente (sin emplear el recurso PAL-24) o realizando alguna consulta telefónica al PAL-24. Se contabilizaron un total de 151 consultas, 74 al PAL-24 y 77 en el HRyC.

De las consultas urgentes registradas en el PAL-24, el motivo más frecuente fue la disnea, seguida del dolor y agitación; otros motivos fueron el sangrado, los vómitos y el bajo nivel de consciencia. Los resultados en relación con las visitas al HRyC fueron similares, siendo el motivo principal la disnea, seguida de dolor, empeoramiento del estado general, astenia, sangrado y caídas (Tabla I).

En cuanto a los datos sociodemográficos, se observó que el $53,8 \%$ eran mujeres, con una media de edad de 78,8 años (siendo la edad máxima 98 años y la mínima 42). La enfermedad de base más frecuente fue el carcinoma de pulmón, seguido del cáncer colorrectal y urológico. Las enfermedades no oncológicas representaron menos de un cuarto del total, siendo la más frecuente la demencia, seguida de la insuficiencia cardiaca y la esclerosis lateral amiotrófica (Figuras 1 y 2 ).

En la primera visita domiciliaria los principales síntomas que cabe destacar fueron la anorexia y el dolor en casi la mitad de los pacientes, seguidos de astenia, disnea, insomnio y estreñimiento (Tabla II).

La mayoría de las consultas urgentes se realizaron fuera del horario del ESAD, estando únicamente en horario el 8,1 \% de las realizadas al PAL-24 y el $26,3 \%$ de las efectuadas al servicio de Urgencias.

Con respecto a la intervención llevada a cabo por el PAL24 , en casi la mitad de los casos la consulta fue resuelta por vía telefónica, precisando el resto el traslado de un recurso al domicilio. Fue una minoría la que precisó traslado al Servicio de Urgencias o se gestionó el ingreso en una Unidad de Cuidados Paliativos (Tabla I). Los fármacos más empleados en la resolución telefónica fueron opioides, ansiolíticos y AINE. Tras la llamada al PAL-24, casi la totalidad de los pacientes permaneció en el domicilio, siendo una minoría la que ingresó en el hospital.

En cuanto a las intervenciones realizadas en el Servicio de Urgencias, en la mayoría de los pacientes se realizó extracción sanguínea y en más de la mitad de ellos pruebas de 
Tabla I. Descripción de la intervención llevada a cabo mediante las llamadas al PAL-24 y las visitas a Urgencias del Hospital Ramón y Cajal

\begin{tabular}{|c|c|c|}
\hline & PAL-24 & Urgencias del Hospital Ramón y Cajal \\
\hline Número de consultas & 74 & 77 \\
\hline Motivo de consulta & Disnea $(29,7 \%)$, dolor (12,2 \%) y agitación $(12,2 \%)$ & $\begin{array}{l}\text { Disnea }(30,3 \%) \text {, dolor }(18,4 \%) \text { y } \\
\text { deterioro del estado general }(15,8 \%)\end{array}$ \\
\hline $\begin{array}{l}\text { Horario } \\
\text { Laborable } 8-15 \mathrm{~h} \\
\text { Laborable } 15-21 \mathrm{~h} \\
\text { Laborable } 21-8 \mathrm{~h} \\
\text { Fin de semana/festivo }\end{array}$ & $\begin{array}{l}8,1 \% \\
35,1 \% \\
21,6 \% \\
35,1 \%\end{array}$ & $\begin{array}{c}26,3 \% \\
30,3 \% \\
18,4 \% \\
25 \% \\
\end{array}$ \\
\hline Intervención & $\begin{array}{l}\text { Vía telefónica }(48,6 \%) \\
\text { envío de recurso a domicilio }(40,5 \%) \\
\text { derivación hospitalaria }(9,5 \%) \text { e ingreso en UCP }(1,4 \%)\end{array}$ & $\begin{array}{l}\text { Analítica }(81,6 \%) \\
\text { Radiografías }(60,5 \%) \\
\text { Ecografía }(1,3 \%) \\
\text { Tomografía computarizada }(1,3 \%) \\
\text { Transfusión de sangre }(7,9 \%) \\
\text { Colocación de sonda nasogástrica }(5,3 \%) \\
\text { Intervención quirúrgica }(2,6 \%)\end{array}$ \\
\hline Fármacos utilizados & $\begin{array}{l}\text { Fármacos utilizados en la resolución telefónica: } \\
\text { Opioides }(27 \%), \\
\text { Ansiolíticos }(13,5 \%) \\
\text { AINE }(9,5 \%) \\
\text { Neurolépticos }(6,8 \%)\end{array}$ & $\begin{array}{l}\text { Fármacos utilizados en Urgencias: } \\
\text { Antibióticos }(32,9 \%) \\
\text { Opioides }(27,6 \%) \\
\text { Corticoides }(21,1 \%)\end{array}$ \\
\hline Resolución & $\begin{array}{l}\text { Permanencia en domicilio }(82,4 \%) \\
\text { Ingreso hospitalario }(6,8 \%) \\
\text { Muerte }(6,8 \%) \\
\text { Ingreso en UCP }(4,1 \%)\end{array}$ & $\begin{array}{l}\text { Alta a domicilio }(55,3 \%) \\
\text { Ingreso en hospitalización }(38,2 \%) \\
\text { Muerte }(6,6 \%)\end{array}$ \\
\hline
\end{tabular}

AINE: antiinflamatorios no esteroideos. UCP: Unidad de Cuidados Paliativos.

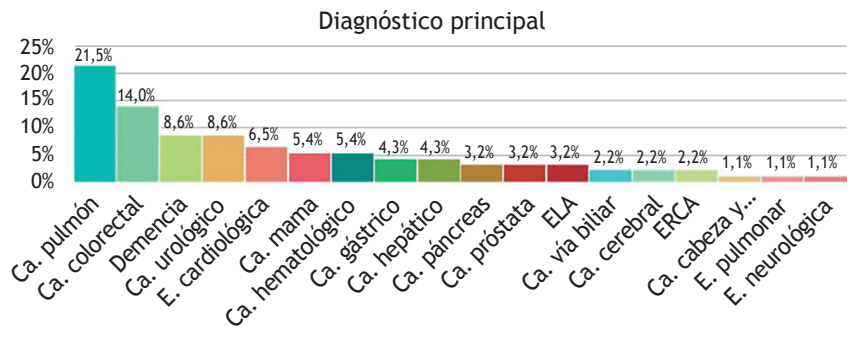

Figura 1. Diagnóstico principal.

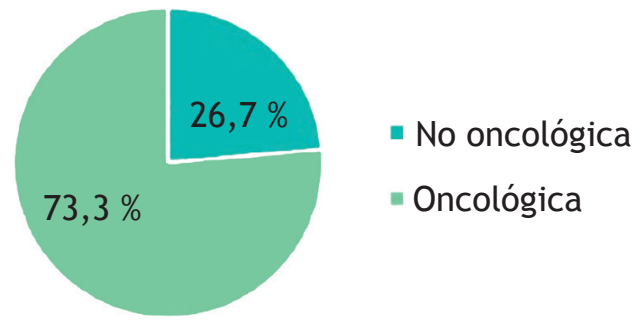

Figura 2. Naturaleza de la patología de base.

imagen (radiografía, ecografía o tomografía computarizada). Las técnicas más realizadas fueron la colocación de sonda nasogástrica o intervenciones quirúrgicas urgentes. Los fármacos más prescritos fueron los antibióticos, en un tercio de la muestra, seguidos de opioides, corticoides y transfusión de sangre, y menos frecuentes fueron los diuréticos, AINE y ansiolíticos. Con respecto a la resolución de la consulta tras la visita a Urgencias, más de la mitad fueron dados de alta
Tabla II. Síntomas presentes en la primera valoración en el domicilio (\%)

\begin{tabular}{lc}
\hline Anorexia & 45,2 \\
Dolor & 43 \\
Astenia & 40,9 \\
Disnea & 28 \\
Insomnio & 25,8 \\
Estreñimiento & 24,7 \\
Depresión & 21,5 \\
Ansiedad & 18,3 \\
Caquexia & 18,3 \\
Náuseas/vómitos & 12,9 \\
Tos & 11,8 \\
Somnolencia & 10,8 \\
Agitación & 8,6 \\
Edemas & 7,5 \\
Diarrea & 5,4 \\
Ascitis & 5,4 \\
Disfagia & 3,2 \\
Xerostomía & 2,2 \\
\hline
\end{tabular}

a su domicilio nuevamente, un tercio ingresó en planta de hospitalización y el resto falleció en el Servicio de Urgencias (Tabla I). 
Del total de pacientes, fallecieron $76(81,7 \%)$ durante el periodo de seguimiento de 3 meses. Un tercio de ellos $(32,3 \%)$ lo hizo en el domicilio, seguido de Unidades de Cuidados Paliativos (21\%), plantas de hospitalización del HRyC $(20,4 \%)$ y, en último lugar, el Servicio de Urgencias de dicho hospital, con un $7,5 \%$ del total.

\section{Discusión y conclusiones}

El número de pacientes derivados al equipo del ESAD del Área Este de Madrid fue de 279, cifra que se acerca a los resultados publicados en el original de la Gaceta Sanitaria ${ }^{5}$ de 2011, donde se estima que la prevalencia de los cuidados paliativos y de soporte en España se situó en torno a tres casos por cada 1000 habitantes, siendo mayor en las poblaciones más envejecidas. Teniendo en cuenta que la población del Área Este de Madrid se compone de unos 530.000 habitantes, de los cuales el $14 \%$ de ellos son mayores de 65 $a^{n}{ }^{6}{ }^{6}$, consideramos importante resaltar el gran porcentaje de población susceptible de cuidados paliativos y las necesidades crecientes de los mismos, dado el envejecimiento poblacional y el aumento en la esperanza de vida.

Las consultas realizadas se centraron en el mal control sintomático, fundamentalmente del dolor y la disnea, encontrándose también dichos síntomas entre los más prevalentes en la primera visita al domicilio. Además, tanto el dolor como la disnea son síntomas que pueden manifestarse en forma de episodios irruptivos, que generan en el paciente y en la familia la necesidad de solicitar una ayuda urgente. Por último, también consideramos que los síntomas están muy relacionados con los principales diagnósticos de los pacientes estudiados, fundamentalmente cáncer de pulmón y de colon. Estos datos coinciden con otros estudios realizados previamente, como el trabajo de Nordly y cols., publicado en $2014^{7}$

La mayoría de las derivaciones fueron por patología oncológica, como cabía esperar; sin embargo, nos llamó la atención el reducido número de pacientes con enfermedad renal crónica avanzada en comparación con estudios previos, como el publicado en 2014 por Teruel y cols. ${ }^{8}$, donde obtuvieron una muestra de 50 enfermos con esta enfermedad en un periodo de seguimiento de 2 años. El motivo de estas diferencias puede subyacer en que la atención sanitaria en este perfil de pacientes es llevada a cabo, en ocasiones, por Atención Primaria en colaboración con Servicios de Nefrología. En cualquier caso, nos deja la puerta abierta a la reflexión, concluyéndose en este mismo estudio que el soporte de la Unidad de Cuidados Paliativos y del Servicio de Nefrología en el paciente con enfermedad renal crónica avanzada no candidato a diálisis puede ser controlado en su domicilio, lo que genera beneficios a muchos niveles.

Dentro de la patología no oncológica derivada al ESAD la más frecuente fue la demencia, seguida de la insuficiencia cardiaca y la esclerosis lateral amiotrófica. Un valor similar se obtuvo en el reciente estudio de una Unidad de Cuidados Paliativos publicado en 2017 por Lo y cols. ${ }^{9}$, con un tamaño muestral de 780 pacientes, y en el que solo el $4 \%$ de la muestra padecía una demencia avanzada.

En los resultados llama la atención que la mayoría de las consultas se realizan fuera del horario de atención de ESAD. Múltiples estudios determinan las ventajas obtenidas de un buen programa de cuidados paliativos en relación con la calidad de vida percibida por el paciente, con una reducción del número de hospitalizaciones, así como de la carga del cuidador ${ }^{10}$. Todo ello nos hace pensar que ampliando el horario de atención domiciliaria podría disminuir el número de consultas y, en muchos casos, las hospitalizaciones, mejorando la calidad de vida de nuestros pacientes. En la revisión publicada en 2015 por Mellar y cols. y llevada a cabo en el Hospital General de Massachusetts ${ }^{10}$, se ratifican estos resultados, resaltando, sin embargo, la necesidad de estudios mejor diseñados que determinen el momento óptimo de intervención, así como el mejor modelo de cuidado. En la revisión sistemática publicada en $2016^{7}$ por Nordly y cols., denuncian la falta de ensayos clínicos centrados en el control sintomático, sobre todo de dolor y disnea, en el ámbito de los cuidados paliativos domiciliarios.

El lugar más prevalente donde se produjo el fallecimiento fue el domicilio, en un tercio de los pacientes; sin embargo, un porcentaje no despreciable de la muestra fallece durante un ingreso hospitalario, en el Servicio de Urgencias o en una Unidad de Cuidados Paliativos, dato que contrasta con lo publicado hasta el momento ${ }^{11}$. Consideramos que este punto puede estar en relación con la necesidad de disponer de un buen soporte familiar que permita garantizar unos cuidados óptimos en el domicilio. Teniendo en cuenta el área de población que abarca nuestro estudio y nuestra experiencia en dicho entorno laboral, son muchas los domicilios donde el único cuidador es un familiar de edad avanzada y con pluripatología, así como otros familiares con horarios laborales complejos.

En cuanto a las limitaciones del estudio, al tratarse de un estudio observacional, descriptivo y prospectivo en una cohorte de pacientes con enfermedad avanzada, pueden existir sesgos de información, siendo los informadores terceras personas en muchas ocasiones. Lo mismo ocurre en relación con los archivos del PAL-24, ya que no siempre existe un registro informático o en papel de la totalidad de las actividades realizadas por ellos, fundamentalmente una vez que se produce el fallecimiento, lo que puede suponer una pérdida en la calidad y cantidad de los datos. En definitiva, nuestros datos están limitados a los disponibles en la historia clínica electrónica del paciente, existiendo potencialmente pérdidas y sesgos.

Por otra parte, al tratarse de un estudio unicéntrico, nuestro tamaño muestral es reducido, existiendo además la posibilidad de que los pacientes acudan a centros hospitalarios y Urgencias distintos a los de referencia, con lo que se perdería, en este caso, la información que de estas visitas se deriva. Cabe añadir, por último, que el limitado periodo de seguimiento de 3 meses también ha podido conducir a la pérdida de datos.

Respecto a las fortalezas del trabajo, destacamos las implicaciones prácticas de conocer los motivos de demanda urgente de los pacientes seguidos en el domicilio, que reflejan la importancia de la continuidad asistencial, así como la organización de recursos, que permitiría proporcionar una mejor cobertura a nuestros pacientes. La demanda urgente en este perfil de pacientes siempre será atendida de una manera más adecuada por el equipo médico que conozca al paciente.

Para concluir, el principal motivo de consulta urgente de los pacientes en seguimiento por un programa de cuidados paliativos domiciliarios es la disnea, seguido del dolor. Se 
trata de una población con diagnóstico de enfermedad oncológica avanzada en su mayoría. Prácticamente la totalidad de las consultas urgentes fueron llevadas a cabo fuera del horario asistencial del ESAD. La mitad de las consultas realizadas al recurso PAL-24 fueron resueltas telefónicamente, y el resto de los casos precisó de un recurso a domicilio. De los pacientes que consultaron directamente al Servicio de Urgencias, la mitad fueron dados de alta de nuevo al domicilio y, del total de pacientes en seguimiento, un tercio de ellos finalmente falleció en el domicilio.

\section{Conflicto de intereses}

Sin conflictos de interés.

Presentado como Trabajo de Fin de Máster (Máster en Cuidados Paliativos y Tratamiento del Paciente con Cáncer Avanzado), Universidad Autónoma de Madrid.

\section{Bibliografía}

1. Espinosa J. Equipos de soporte domiciliario de cuidados paliativos en España. Med Clin (Barc). 2010;135:470-5.

2. Consejería de Sanidad y Consumo. Plan integral de cuidados paliativos de la Comunidad de Madrid 2005-2008. Junio 2005. Disponible en: http://bit.ly/1MTknuz
3. Smith AK, McCarthy E, Weber E, Cenzer IS, Boscardin J, Fisher $J$, et al. Half of older Americans seen in emergency department in last month of life; most admitted to hospital, and many die there. Health Aff (Millwood). 2012;31:1277-85.

4. McNamara BA, Rosenwax LK, Murray K, Currow DC. Early admission to community-based palliative care reduces use of emergency departments in the ninety days before death. J Palliat Med. 2013;16:774-9.

5. Vega T, Arrieta E, Lozano JE, Miralles M, Anes Y, Gómez C, et al. Atención sanitaria paliativa y de soporte de los equipos de atención primaria en el domicilio. Gac Sanit. 2011;25:205-10.

6. Memoria 2006. Hospital Universitario Ramón y Cajal. Disponible en: http://www.hrc.es/info/memoria2006/influencia2.htm

7. Nordly M, Vadstrup E, Sjøgren P, Kurita GP. Home-based specialized palliative care in patients with advanced cancer: A systematic review. Palliat Support Care. 2016;14:713-24.

8. Teruel JL, Rexach L, Burguera V, Gomis A, Rodríguez-Mendiola $\mathrm{N}$, Díaz A, et al. Programa de atención domiciliaria a pacientes con enfermedad renal crónica avanzada. Experiencia de dos años. Nefrología. 2014;34:611-6.

9. Lo AT, Karuza J, Berall A, Perri GA. Prevalence of dementia in a Geriatric Palliative Care Unit. Am J Hosp Palliat Care. 2018; 35:799-803.

10. Davis MP, Temel JS, Balboni T, Glare P. A review of the trials which examine early integration of outpatient and home palliative care for patients with serious illnesses. Ann Palliat Med. 2015;4:99-121.

11. Freeman S, Smith TF, Neufeld E, Fisher K, Ebihara S. The wish to die among palliative home careclients in Ontario, Canada: A cross-sectional study. BMC Palliat Care. 2016;15:24. 